\title{
Piezoelectric Screening in Single InAs/AIAs Quantum Dots
}

D. Sarkar, H. P. van der Meulen, J. M. Calleja, J. M. Meyer, R. J. Haug, and K. Pierz

Citation: AIP Conference Proceedings 1199, 299 (2010); doi: 10.1063/1.3295419

View online: https://doi.org/10.1063/1.3295419

View Table of Contents: http://aip.scitation.org/toc/apc/1199/1

Published by the American Institute of Physics 


\title{
Piezoelectric Screening in Single InAs/AIAs Quantum Dots
}

\author{
D. Sarkar ${ }^{1}$, H. P. van der Meulen ${ }^{1}$, J. M. Calleja ${ }^{1}$, J. M. Meyer ${ }^{2}$, R. J. Haug ${ }^{2}$ and K. \\ Pierz ${ }^{3}$
}

${ }^{1}$ Departamento de Física de Materiales, Universidad Autónoma de Madrid, E-28049 Madrid, Spain
${ }^{2}$ Institut für Festkörperphysik, Leibniz Universität Hannover, D-30167 Hannover, Germany
${ }^{3}$ Physikalisch-Technische Bundesanstalt Braunschweig, D-38116 Braunschweig, Germany

\begin{abstract}
Micro-photoluminescence spectroscopy for varying temperature, excitation intensity and energy was performed on a single InAs/AlAs self-assembled quantum dot. A broad sideband at $1.2 \mathrm{meV}$ of the zero phonon line is attributed to the exciton-acoustic phonon interaction via deformation potential coupling. The piezoelectric coupling of the exciton to long-wavelength phonons is also observed and gives rise to a narrow low-energy sideband at about 0.25 $\mathrm{meV}$ of the zero-phonon line. This band strengthens with decreasing density of photocreated free carriers, since they screen out the piezoelectric coupling mechanism.
\end{abstract}

Keywords: Photoluminescence, III-V semiconductors, quantum dots, exciton-phonon interaction, piezoelectricity. PACS: 78.67.Hc, 78.55.Cr, 63.22.-m, 63.20.kk

\section{INTRODUCTION}

The coherence properties of the confined carriers and excitations in semiconductor quantum dots (QDs) play a crucial role in quantum information applications. The phase coherence can be lost either by population relaxation processes or by coupling to acoustic phonons (pure dephasing). The former produces a broadening of the Lorentzian zero-phonon line (ZPL). Coupling to acoustic phonons gives rise to non-Lorentzian sidebands [1]. Exciton-acoustic phonon interactions have been treated theoretically considering both the deformation potential (DP) and piezoelectric (PZ) coupling mechanisms $[1,2,3]$. In this work we performed micro photoluminescence (PL) spectroscopy on a single InAs/AlAs QD. We observe a narrow low-energy sideband and show that it is originated by the PZ coupling of the exciton to longwavelength acoustical phonons.

\section{EXPERIMENTAL}

The sample was grown by molecular beam epitaxy and self-assembly of InAs dots between $20 \mathrm{~nm}$ thick AlAs layers. A $10 \mathrm{~nm}$ thick GaAs cap layer was deposited on top of the sample. Substrate rotation was interrupted during the InAs deposition in order to obtain a QD density gradient. Metal masks on top of the sample with square apertures ranging from 0.2 to
$10 \mu \mathrm{m}$ allow for single QD spectroscopy at low density sites.

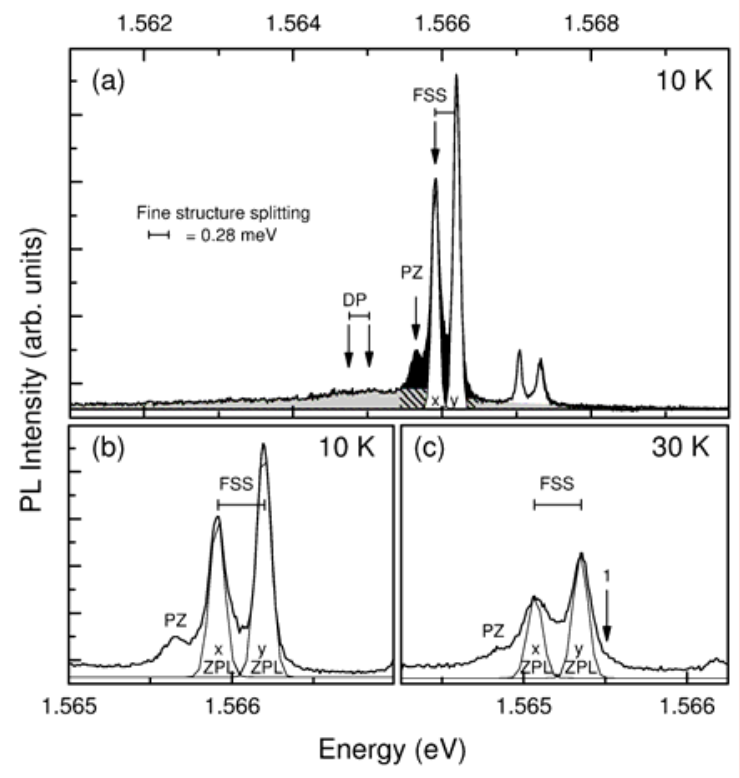

FIGURE 1. a) PL spectrum of a single QD. The doublet is composed of a central Lorentzian ZPL and asymmetric acoustic phonon wings. b) $(10 \mathrm{~K})$ : The narrow $\mathrm{PZ}$ sideband of the x-peak is observed $0.2 \mathrm{meV}$ below the ZPL. c) $(30 \mathrm{~K})$ : by increasing the temperature, sidebands also appear on the high energy sides of the y-ZPL (arrow 1). 
The sample was mounted in a continuous-flow cryostat at $10 \mathrm{~K}$. A microscope objective with numerical aperture of 0.55 focused the Argon and Ti:Sapphire lasers on a $\mu \mathrm{m}$-sized spot and also collected the signal. Detection was done with a $\mathrm{LN}_{2}$ cooled Si-CCD mounted on a $0.85 \mathrm{~m}$ double grating spectrometer.

\section{RESULTS AND DISCUSSION}

The PL spectrum of the exciton transition consists of a fine-structure split doublet of linearly crosspolarized peaks $x$ and $y$ (Fig. 1a). The large finestructure splitting of $0.28 \mathrm{meV}$ is due to the high AlAs barriers [4]. At $10 \mathrm{~K}$ we observe broad acoustic phonon wings (light grey area) corresponding to the DP mediated exciton-acoustic phonon interaction.

Narrow low energy sidebands (black areas in fig. 1a) are observed close to the ZPL. We attribute them to the interaction of the exciton with long wavelength acoustic phonons mediated by PZ coupling. They are shown in detail in Fig. $1 \mathrm{~b}$ and c. At $10 \mathrm{~K}$ we observe an additional narrow low-energy band located at 0.2 meV below the $\mathrm{x}-\mathrm{ZPL}$ (marker PZ). At higher temperature $(30 \mathrm{~K})$, a wing appears on the high energy side of the $y-Z P L$ (arrow 1). Both DP and PZ sidebands evolve from asymmetric at low temperature to symmetric bands.

For decreasing excitation power the acoustic phonon wings become wider and more pronounced while the ZPLs lose weight compared to the sidebands (Fig. 2). For the intensities below $P_{0} / 16$ the ZPLs are not even observed any longer and the transition lineshape is completely dominated by the PZ sidebands. This power dependence is indicative of screening. In contrast to the DP coupling mechanism, PZ coupling can be screened out by free carriers [2, 3]. By increasing the excitation power, more free carriers are photo-generated and consequently the PZ mediated exciton-acoustic phonon interaction weakens. When exciting above the wetting layer absorption edge (right panel in Fig.2) the screening is even more efficient.

The strong PZ effects in our dots compared to previously reported experiments, where the DP coupling dominates are due to the role of the barrier material. In the models presented so far the elastic properties of dot and barrier material are taken equal $[1,3,5]$, whereas the PZ coefficient of AlAs $\left(e_{14}=\right.$ $\left.0.23 \mathrm{C} / \mathrm{m}^{2}\right)$ is large compared to $\mathrm{GaAs}(-0.16)$ and InAs (-0.045) [6]. Al intermixing into the dot might also give large PZ effects. Besides, recent studies have pointed out the importance of second order contributions to the PZ field which are very sensitive to QD shape, size and composition [7, 8]. The QD presented here belongs to the large size fraction of our
QD distribution. These large QDs should have a larger aspect ratio [9] giving rise to larger PZ effects [8]. A more detailed study will be published elsewhere [10].

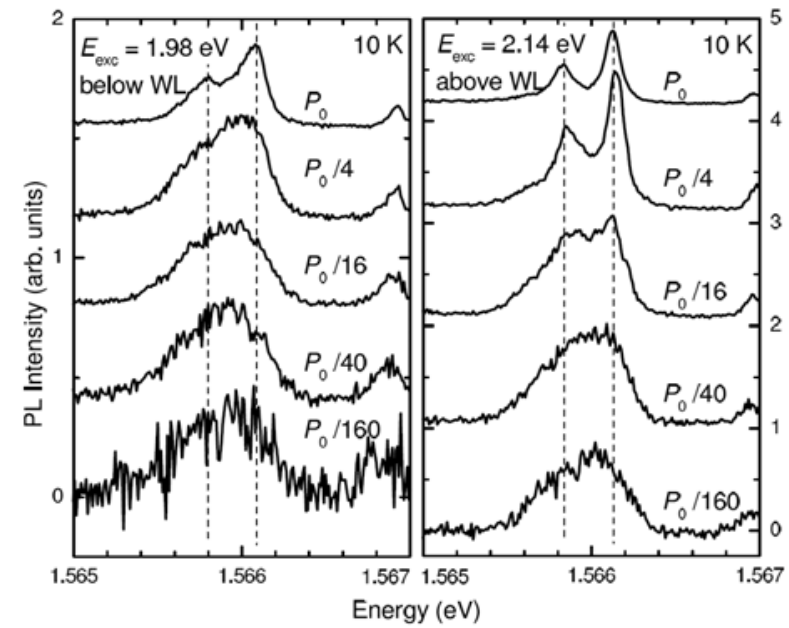

FIGURE 2. PL spectra of the exciton for a series of excitation power $\left(P_{0}=0.4 \mathrm{~mW}\right)$. For increasing excitation intensities the sidebands get less pronounced opposite to the ZPL, which gains weight. This indicates a screening effect of the acoustic phonon-exciton coupling.

In summary, InAs QDs with an AlAs-barrier show strong piezoelectric effects. The interaction can be screened be photo-created carriers, and this switching ability opens the possibility to control dephasing rates, which may be used in future quantum devices.

\section{ACKNOWLEDGMENTS}

This work has been supported by research contracts of the Spanish Ministry of Education (MEC MAT2005-01388, NAN2004-09109-C04-04, TEC2004-05260-C02-02, Consolider CSD 2006-19) and the Community of Madrid (CAM S-0505-ESP0200).

\section{REFERENCES}

1. L. Besombes et al., Phys. Rev. B 63, 155307 (2001).

2. C. B. Duke, and G. D. Mahan, Phys. Rev. 139, A1965 (1965).

3. T. Takagahara, Phys. Rev. B 60, 2638 (1999).

4. D. Sarkar et al., J. Appl. Phys. 100, 023109 (2006).

5. E. Peter et al., Phys. Rev. B 69, 041307 (2004).

6. S. Adachi, Properties of Group-IV, III-V and II-VI Semiconductors, John Wiley and Sons, 2005, ISBN 0470090324.

7. G. Bester et al., Phys. Rev. B 74, 081305 (2006).

8. A. Schliwa et al., Phys. Rev. B 76, 205324 (2007).

9. P. Ballet et al., Appl. Phys. Lett. 75, 337 (1999).

10. D. Sarkar et al., to be published. 